\title{
Temozolomide in treatment resistant prolactinomas
}

\author{
Shree V.N, Datuk Dr Mohamed Badrulnizam L.B
}

Hospital Putrajaya, Malaysia

\section{Abstract}

We report a case of a 23-year-old gentleman, diagnosed with macroprolactinoma at age 14 . He presented with bitemporal hemianopia and no other symptoms of prolactinoma. He underwent two trans-sphenoidal surgeries and subsequently developed panhypopituitarism requiring replacement therapy. Despite compliance and up titrated doses of cabergoline (maximum total dose of $12 \mathrm{mg} /$ week), resistant prolactin (PRL) levels up to $22614 \mathrm{mU} / \mathrm{L}$ were noted. Trial of temozolomide combined with cabergoline showed prolactin levels that drastically declined over 16 weeks and remained stable at 52 weeks with significant radiological remission.

Key words: Resistant prolactinoma, temozolomide, cabergoline

\section{Introduction}

Prolactinomas are amenable to medical treatment alone using dopamine agonists, with high probability of disease control with endocrine and radiographic remission. Prolactinomas account for $40-50 \%$ of pituitary adenomas. However, these tumours occasionally exhibit aggressive behaviours that do not respond to standard treatments of medical therapy, surgical resection, and adjuvant radiation [2]. In cases of treatment resistance despite high doses of dopamine agonists, studies show significant remission with adjuvant chemotherapy with temozolomide (TMZ).

\section{Case Presentation}

panhypopituitarism after the first transsphenoidal surgery requiring hormone replacement with Levothyroxine $100 \mathrm{mcg}$ once daily, Hydrocortisone $10 \mathrm{mg}$ twice daily and
IM testosterone $250 \mathrm{mcg}$ three weekly. Patient was commenced on cabergoline, with prolactin levels reflecting progressive improvement. However, prolactin levels were detected to be increasing and was attributed to poor compliance. The importance of compliance was reinforced A 23-year-old gentleman of Chinese descent presented at the age of 14 years with features of bitemporal hemianopia at a private health institution. He underwent MRI imaging which confirmed the diagnosis of a pituitary macroadenoma and the initial prolactin levels were documented as $8800 \mathrm{mU} / \mathrm{L}$. He underwent transsphenoidal surgical resection of the tumour at age 14 and a repeat surgery at 16 years of age. He developed however despite good compliance and up titration of cabergoline doses (total dose of $12 \mathrm{mg} /$ week), prolactin levels continued to increase exponentially. The highest prolactin levels recorded was $22,614 \mathrm{mU} / \mathrm{L}$. Despite elevated prolactin levels, patient remained asymptomatic and did not

Correspondence email: vidhya.nagendram@gmail.com

iD https://orcid.org/0000-0002-0811-2168 
experience galactorrhoea. Serial MRIs done, revealed residual, now progressing to become treatment resistant macroprolactinoma. Patient had declined any further surgical intervention. As a consequence of treatment resistance with maximum tolerated dose of dopamine agonist, the cytotoxic alkylating agent, Temozolomide was considered as neo-adjuvant therapy. Oral Temozolomide was commenced in November 2016 and was prescribed at a dose of $300 \mathrm{mg}$ daily for day 1 till day 5 of each month cycle (150 to $200 \mathrm{mg} / \mathrm{m}^{2}$ of body surface/ day during 5 days every 28 days cycle). Temozolomide was added on concurrently with cabergoline $1.5 \mathrm{mg}$ daily. Baseline complete blood count and liver function test was done prior to commencement. These blood parameters were then subsequently repeated along with prolactin levels prior to each cycle of Temozolomide to monitor treatment efficacy and for surveillance of occurrence of adverse reactions to the drug. Prolactin levels drastically declined over 16 weeks and remained stable at 52 weeks (Figure 1 and Table 1). Both Temozolomide and cabergoline $(0.5 \mathrm{mg} /$ week $)$ were down titrated. Radiological remission of prolactinoma evident on serial MRI. (Image $1 \& 2$ ).

\section{Table 1. Laboratory results comparing baseline values and at 52 weeks.}

\begin{tabular}{lllll}
$\begin{array}{l}\text { Laboratory } \\
\text { Investigations }\end{array}$ & $\begin{array}{l}\text { Values at baseline } \\
\text { /November 2016 }\end{array}$ & $\begin{array}{l}\text { Values at week 52 } \\
\text { (December 2017) }\end{array}$ & Ref. range & Units \\
\hline Prolactin & 4458 & 393 & $98-456$ & $\mathrm{mU} / \mathrm{L}$ \\
TSH & 0.06 & 0.16 & $0.23-4.2$ & $\mathrm{pmol} / \mathrm{L}$ \\
T4 & 17.3 & 8.7 & $12-22$ & $\mathrm{mU} / \mathrm{l}$ \\
Cortisol & 19.5 & $<13$ & $145-619$ & $\mathrm{nmol} / \mathrm{L}$ \\
FSH & $<0.30$ & & $0.17-9.61$ & $\mathrm{U} / \mathrm{L}$ \\
LH & $<0.50$ & & $0.07-8.52$ & $\mathrm{U} / \mathrm{L}$ \\
Serum testosterone & 31 & $9.9-27.8$ & $\mathrm{nmol} / \mathrm{L}$ \\
\hline
\end{tabular}

\section{Fig.1 Prolactin levels}

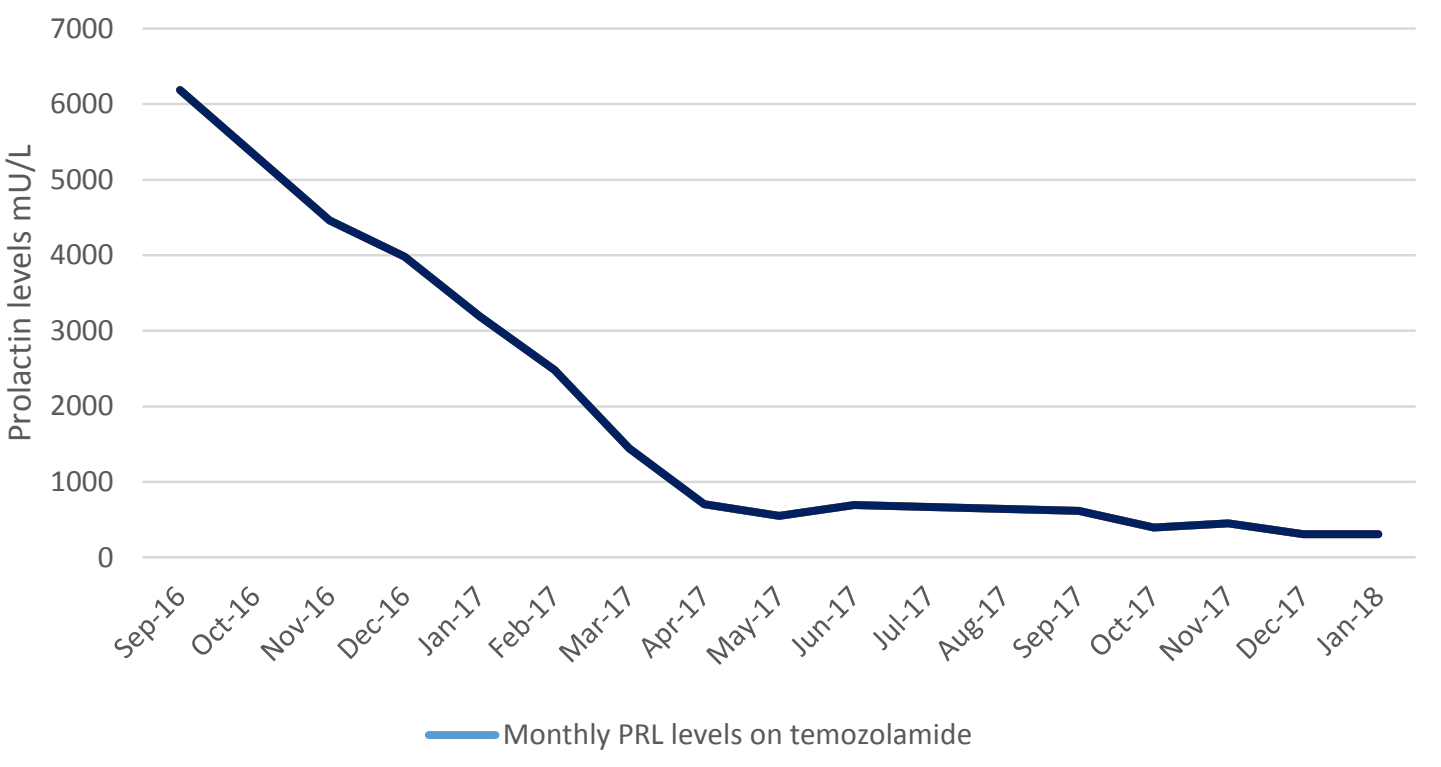




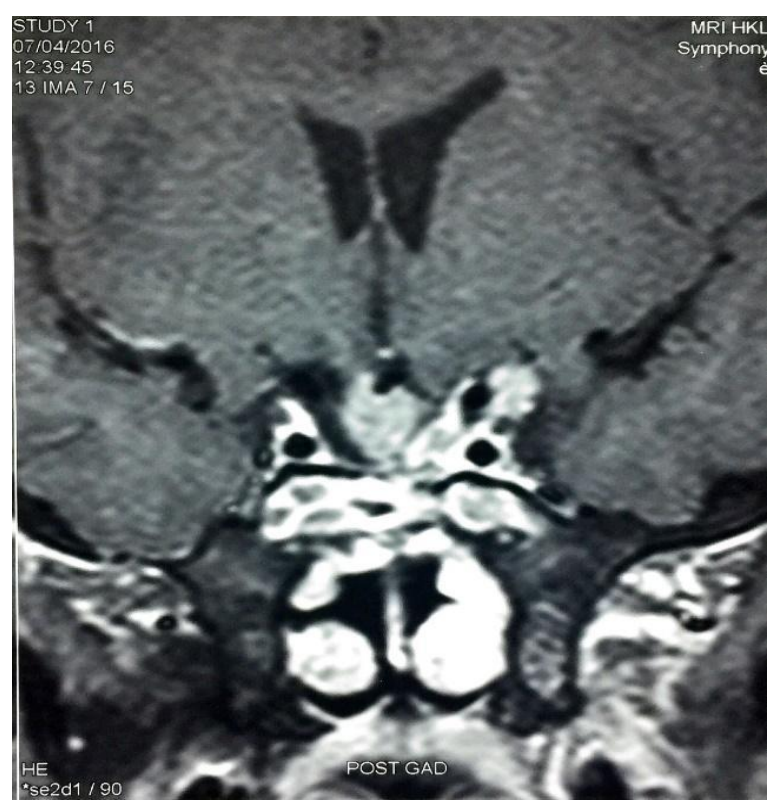

Image 1. Contrast-enhanced coronal T-1 weighted MRI showing remnant sellar tumour post-surgical resection in 2016.

\section{Discussion}

Temozolomide is an oral alkylating cytotoxic drug that crosses blood brain barrier and is established in treating high-grade gliomas. It was first used to treat glioblastoma in cases resistant to standard therapy in 1992 (4). Later, the use of temozolomide became the first-line treatment against glioblastoma and expanded to astrocytoma, gliomas, advanced melanomas, and neuroendocrine tumours (5). Temozolomide as salvage therapy to treat aggressive pituitary carcinomas appeared in 2004. (3) Pharmacology of temozolomide identifies that it rapidly converts into active 5-(3-methyl-triazeno)-imidazole-4-carboxamide

responsible for DNA lesions by binding methyl groups at O6-guanine leading to DNA mismatch and cell apoptosis (6). Methylguanine methyltransferase (MGMT) is a DNA repair enzyme, which counteracts the chemotherapeutic effect of temozolomide, thus low MGMT levels show favourable response to temozolomide.

The common adverse effects of temozolomide include lethargy, dizziness, nausea, vomiting, headaches and diarrhoea. Most of them are transient and tolerable and seldom lead to treatment cessation, thus temozolomide can be used for a long-term treatment. Hematologic toxicities

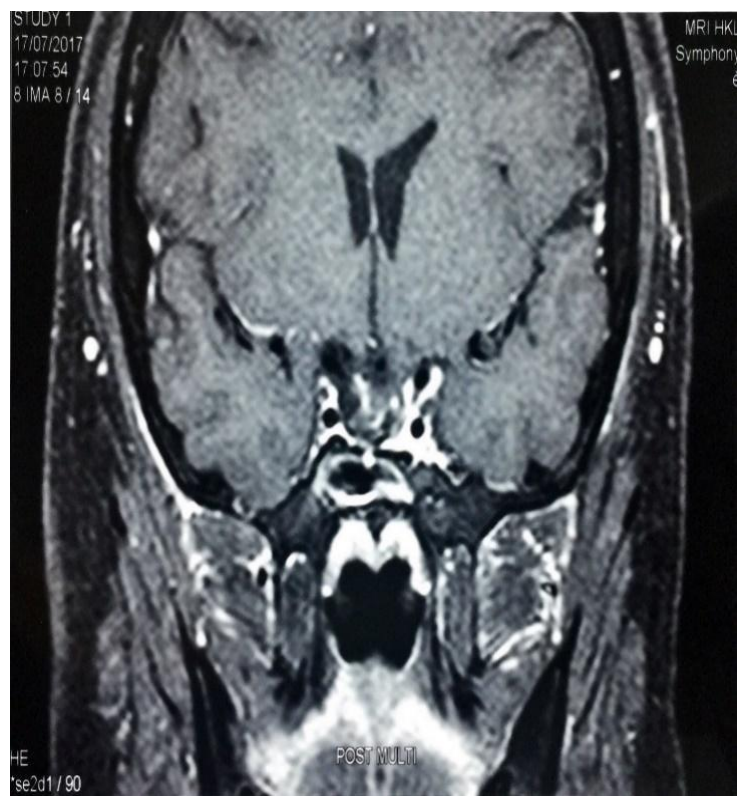

Image 2. Contrast-enhanced coronal T-1 weighted MRI showing significant remission in lesion 52 weeks after neo-adjuvant therapy with temozolamide.

such as thrombocytopenia, leukocytopenia, and lymphocytopenia are also noticed in some cases and these infrequent side effects usually lead to the cessation of temozolomide treatment or a reduction in the temozolomide dose or an increase in the interval between cycles.

Current clinical practice supports the use of temozolomide as a salvage therapy for malignant and highly aggressive prolactinomas, which have failed to respond to dopamine agonist therapy, multiple surgical interventions and radiotherapy. Despite limited clinical data on the long-term side effects of temozolomide, nevertheless in selected cases, consideration should be given to using temozolomide earlier in the treatment algorithm to prevent repeated surgical interventions or radiotherapy related adverse effects to achieve endocrine and radiological remission of prolactinomas.

\section{Conclusion}

We presented a case of a patient accordant with treatment resistant prolactinoma. Despite having had two transsphenoidal surgeries and maximum tolerated dopamine agonist only responded to neo-adjuvant therapy with Temozolomide. 
The 2011 Endocrine Society Guidelines on the management of hyperprolactinaemia recommends consideration of temozolomide for treatment resistance prolactinomas (3). However, despite a decade of use, clinical data is limited about temozolomide efficacy for aggressive pituitary adenomas and pituitary carcinomas. Multi-centre research is crucial to provide more therapy strategies and establish clinical guidelines for earlier diagnosis and treatment with neo-adjuvant drugs for these refractory tumours.

\section{Conflict of Interest}

The authors declare that there are no conflicts of interest regarding the publication of this paper.

References

1. Guidelines of the Pituitary Society for the diagnosis and management of prolactinomas. Casanueva FF, Molitch ME, Schlechte JA, et al. Clin Endocrinol (Oxf) 2006;65:265-273

2. Fadul, C.E., Kominsky, A.L., Meyer, L.P. et al. (2004) Pituitary carcinomas respond to temozolomide. Journal of NeuroOncology,6, 374

3. Melmed, S., Casanueva, F.F., Hoffman, A.R.etal.(2011) Diagnosis and treatment of hyperprolactinemia: an Endocrine Society clinical practice guideline. Journal of Clinical Endocrinology and Metabolism

4. O’Reilly SM, Newlands ES, Glaser MG, et al. Temozolomide: a new oral cytotoxic chemotherapeutic agent with promising activity against primary brain tumours. Eur J Cancer 1993;29A:940-2.

5. Treatment of aggressive prolactinoma with temozolomide, A case report and review of literature up to date Cheng Chen, MD, ${ }^{a}$

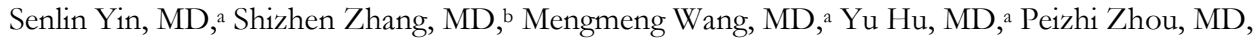

6. Sheehan J, Rainey J, Nguyen J, et al. Temozolomide-induced inhibition of pituitary adenoma cells. J Neurosurg 2011;114:354 8. 